\title{
Neural stem cell transplantation improves learning and memory by protecting cholinergic neurons and restoring synaptic impairment in an amyloid precursor protein/presenilin 1 transgenic mouse model of Alzheimer's disease
}

\author{
QING ZHU $^{1 *}$, NIANPING ZHANG ${ }^{2 *}$, NAN HU ${ }^{1}$, RONGRONG JIANG $^{1}$, \\ HUICONG LU ${ }^{1}$, AIGUO XUAN ${ }^{3}$, DAHONG LONG ${ }^{3}$ and YAN CHEN ${ }^{1}$

\begin{abstract}
${ }^{1}$ Department of Rehabilitation Medicine, The Second Affiliated Hospital of Guangzhou Medical University, Guangzhou, Jinan, Shandong 250355; ${ }^{3}$ Department of Human Anatomy, Guangzhou Medical University, Guangzhou,
\end{abstract} \\ Guangdong 510260; ${ }^{2}$ The Teaching and Research Section of Surgery, Shandong University of Traditional Chinese Medicine, \\ Guangdong 511436, P.R. China
}

Received January 31, 2019; Accepted September 4, 2019

DOI: $10.3892 / \mathrm{mmr} .2020 .10918$

\begin{abstract}
Alzheimer's disease (AD) is the most prevalent age-related neurodegenerative disorder. It is featured by the progressive accumulation of $\beta$-amyloid $(\mathrm{A} \beta)$ plaques and neurofibrillary tangles. This can eventually lead to a decrease of cholinergic neurons in the basal forebrain. Stem cell transplantation is an effective treatment for neurodegenerative diseases. Previous studies have revealed that different types of stem or progenitor cells can mitigate cognition impairment in different Alzheimer's disease mouse models. However, understanding the underlying mechanisms of neural stem cell (NSC) therapies for $\mathrm{AD}$ requires further investigation. In the present study, the effects and the underlying mechanisms of the treatment of AD by NSCs are reported. The latter were labelled with the enhanced green fluorescent protein (EGFP) prior to implantation into the bilateral hippocampus of an amyloid precursor protein (APP)/presenilin 1 (PS1) transgenic (Tg) mouse model of AD. It was observed that the number of basal forebrain cholinergic neurons was restored and the expression of choline acetyltransferase (ChAT) protein was increased. Moreover, the levels of synaptophysin (SYP), postsynaptic density protein 95 (PSD-95) and microtubule-associated protein (MAP-2) were significantly increased in the hippocampus of NSC-treated
\end{abstract}

Correspondence to: Professor Yan Chen, Department of Rehabilitation Medicine, The Second Affiliated Hospital of Guangzhou Medical University, 250 Changgang East Road, Guangzhou, Guangdong 510260, P.R. China

E-mail: chenyandoctor@163.com

*Contributed equally

Key words: Alzheimer's disease, neural stem cell, cholinergic neurons, synapse, transplantation
AD mice. Notably, spatial learning and memory were both improved after transplantation of NSCs. In conclusion, the present study revealed that NSC transplantation improved learning and memory functions in an AD mouse model. This treatment allowed repairing of basal forebrain cholinergic neurons and increased the expression of the cognition-related proteins SYP, PSD-95 and MAP-2 in the hippocampus.

\section{Introduction}

Alzheimer's disease (AD) is the most common neurodegenerative disorder, affecting over 5 million people in the U.S. alone (Alzheimer's Association, 2016). It has become increasingly clear that $\mathrm{AD}$ is associated with multiple causes. In addition to the deposition of $\beta$-amyloid (A $\beta$ ) proteins and neurofibrillary tangles, inflammation and oxidative stresses, metabolic disorders, impaired calcium ion channels, mitochondrial dysfunction and the lack of neurotrophic factors are also linked with its pathomechanism (1). Collectively, these pathologies ultimately lead to the loss of cholinergic neurons and synapses (2). Despite decades of research, there is no effective treatment to cure AD (3).

In recent decades, significant progress has been made in the treatment of neurodegenerative diseases by neural stem cell-based therapy. Neural stem cells (NSCs) have a strong potential of self-renewal and multi-differentiation. Furthermore, they can differentiate into neurons, astrocytes and oligodendrocytes (4). NSC transplantation may be an effective method to cure neurodegenerative diseases by repairing and replenishing functional neurons $(5,6)$. Several studies have revealed that transplanting NSC can alleviate the learning and memory impairment in an $\mathrm{AD}$ mouse model $(7,8)$. It has been revealed that transplanted NSC survived, migrated, and differentiated into neurons (only a small amount). In addition, NSC can secrete neurotrophic factors (9), inhibit inflammation, enhance mitochondrial functions (10) and even promote the activation of endogenous NSCs (11). Unfortunately, 
little is known about the influence of NSC transplantation on cholinergic neurons in the basal forebrain of APP/PS1 transgenic mice.

Cholinergic neurons are closely related to cognition and memory functions. In AD, the loss of cholinergic neurons in the basal forebrain leads to a decrease in the number of cholinergic fibers in the hippocampus and the neocortex (12). Nevertheless, synaptic loss is the principal correlate of disease progression and loss of cholinergic neurons thus causing cognitive deficits (13). However, limited literature has reported the association between neural stem cell transplantation and basal forebrain cholinergic neurons. In the present study, enhanced green fluorescent protein (EGFP)-labeled NSCs were bilaterally transplanted into the hippocampus of 12-month-old APP/PS1 transgenic mice and age-matched wild-type (WT) mice, the effects of neural stem cell transplantation on basal forebrain cholinergic neurons and the recovery of synaptic impairment and its relationship with cognitive functions were investigated.

\section{Materials and methods}

Materials. The following reagents were used in the present study: Dulbecco's modified Eagle's medium/F-12 (1:1) and fetal bovine serum (FBS) were purchased from HyClone; GE Healthcare Life Sciences; B27 supplements and Accutase were obtained from Gibco; Thermo Fisher Scientific; epidermal growth factor (EGF) and basic fibroblast growth factor (b-FGF) were obtained from PeproTech, Inc.; nestin (cat. no. ab6142), $\beta$-tubulin III (cat. no. ab78078), GFAP (cat. no. ab10062), and choline acetyl transferase (ChAT) antibodies (cat. no. ab6168) were purchased from Abcam; postsynaptic density protein 95 (PSD95) (cat. no. 3450), synaptophysin (SYP) (cat. no. 5461), MAP2 (cat. no. 4542), and doublecortin (DCX) (cat. no. 4604) antibodies were obtained from Cell Signaling Technology, Inc.; Alexa Fluor ${ }^{\mathrm{TM}} 568$ goat anti-mouse IgG (cat. no. A-11004) and Alexa Fluor 594 donkey anti-rabbit IgG (cat. no. A32754) were purchased from Thermo Fisher Scientific, Inc.; ChAT antibody (cat. no. DF6964) and HRP-conjugated goat anti-rabbit IgG (cat. no. S0001) were obtained from Affinity Biologicals, Inc.

Animals. In total, 24 APP/PS1 (APPswe, PSEN1dE9) double transgenic mice (age, 5 months; weight, 25-35 g) were obtained from the Experimental Animal Center (Guangdong, China) (certificate no. 44007200038817), and were then maintained in our laboratory (Animal experiment center, Guangzhou Medical University) for another 7 months. In total, three EGFP-labeled mice (age, 50 days; weight, 15-20 g) were provided by the Nanjing Biomedical Research Institute [certificate no. SCXK (SU) 2015-0001]. When the mice suffered trauma, infection and weight loss (>20\%), they were not suitable for the experiment and were euthanized by cervical dislocation. Embryos $(n=8-10)$ of 12.5-14.5 days were used in this study. All animals were kept in separate cages at room temperature $\left(\sim 22-24^{\circ} \mathrm{C}\right)$ with a 12 -h light/dark cycle and free access to water and food. All procedures were approved by the Animal Ethics Committee of the Second Affiliated Hospital of Guangzhou Medical University (certification no. A2018-016).
Preparation of NSCs. NSCs were derived from the embryonic brain (E12.5-14.5 days) of pregnant EGFP-labeled mice. The pregnant mice were fixed in supine position, the abdomen was sterilized twice with iodine, then cut with sterile scissors, and the entire uterus was removed and placed in alcohol. The mouse embryo was washed twice in ethyl alcohol prior to placing it in D-Hank's balanced salt solution. The fetal mouse brain was separated from septal areas, the cerebellum and olfactory bulb were excised, the meninges and blood vessels were stripped, and then washed in cold D-Hank's balanced salt solution. After dissection and digestion, samples were re-suspended in Dulbecco's modified Eagle's medium/F-12 (1:1) (HyClone; GE Healthcare Life Sciences) medium containing $20 \mathrm{ng} / \mathrm{ml}$ of epidermal growth factor (EGF), $20 \mathrm{ng} / \mathrm{ml}$ of basic fibroblast growth factor (b-FGF; both from PeproTech, Inc.) and 2\% B27 (Gibco; Thermo Fisher Scientific, Inc.). Cells (1x10 $/ \mathrm{ml})$ were inoculated into $25-\mathrm{ml}$ culture flasks and maintained at $37^{\circ} \mathrm{C}$ in an incubator with $5 \% \mathrm{CO}_{2}$ and $95 \%$ humidity.

To assess the differentiation ability of NSCs, P2 neurospheres were dissociated with Accutase (Gibco; Thermo Fisher Scientific, Inc.) and inoculated into 24-well plates pre-coated with polylysine (Sangon Biotech, Inc.). When cells adhered closely to the plate, one plate was used to assess stem cell properties. The other was used to investigate the differentiation ability after 5 days with fetal bovine serum (FBS) co-cultured by cell immunofluorescence staining. In short, cells were rinsed three times ( 5 min each time) in phosphate-buffered saline (PBS) solution prior to incubation in $0.3 \%$ Triton $\mathrm{X}-100$ to destabilize cell membranes. Then, the cells were incubated in $5 \%$ bovine serum albumin (BSA) at room temperature for $2 \mathrm{~h}$. Mouse anti-nestin antibody, mouse anti- $\beta$-tubulin III antibody, mouse anti-GFAP antibody (all 1:300, Abcam) were applied and incubated overnight at $4^{\circ} \mathrm{C}$. After washing the primary antibodies, Alexa Fluor 568 goat anti-mouse IgG (1:500) was added and samples were incubated at RT for $2 \mathrm{~h}$. DAPI (Wuhan Boster Biological Technology, Ltd.) staining was then performed for $15 \mathrm{~min}$. Images were captured with the use of a fluorescence microscope (magnification, x200-400).

Surgical transplantation of NSCs into the hippocampus. P2 generation NSCs were used in the experiment. Before transplantation, neurospheres were dissociated with Accutase and washed twice in PBS. Viable cells were counted using trypan blue and the density of living cells was adjusted to $1 \times 10^{5}$ cells $/ \mu 1$ in PBS. Bilateral hippocampal sites were selected for transplantation according to the Mouse Brain Stereotaxic Atlas. Twenty-four 12-month-old male APP/PS1 transgenic mice were randomly divided into two groups: The AD model group (Tg-AD group), the NSC-transplanted group (Tg-NSC group). Twelve wild-type mice of the same age were used as the normal control group (WT group). The WT and Tg-AD group were injected with $5 \mu \mathrm{l}$ PBS in the bilateral hippocampus and the NSC group was injected with $5 \mu 1$ NSC suspension (re-suspended by PBS). In brief, mice were anaesthetized by intraperitoneal injection of $1 \%$ pentobarbital sodium $(50 \mathrm{mg} / \mathrm{kg})$, which was prepared with $0.9 \%$ normal saline and fixed with the use of a stereotaxic apparatus. Each mouse hippocampus was injected bilaterally with $5 \mu \mathrm{l}$ of either vehicle (Tg-AD group and WT group) or NSCs (Tg-NSC group) at a rate of $1 \mu \mathrm{l} / \mathrm{min}$. The injection site was at anteroposterior 
(AP): $-2.06 \mathrm{~mm}$, mediolateral (ML): $\pm 1.85 \mathrm{~mm}$, and dorsoventral (DV): $-2.50 \mathrm{~mm}$ relative to bregma. The needle was maintained in place for an additional $5 \mathrm{~min}$ to allow the liquid to diffuse into the tissue. After injection, the incision was sealed and sterilized prior to placing the mice in single cages.

Morris water-maze $(M W M)$. Ten days after NSC transplantation, Morris water maze (MWM) was used to assess hippocampal-dependent learning and memory. MWM training was comprised of 2 procedures: The place navigation test and the spatial probe test. All the trials were run in a 1-m diameter circular pool, divided into 4 equal quadrants by different symbols. A hidden platform was placed $1.5 \mathrm{~cm}$ underneath the surface of the opaque water. Swimming paths were recorded by a computerized video imaging analysis system. The place navigation test lasted 4 days. Mice were placed into the water quadrants facing the wall of the pool to record the escape latency while searching for the platform for $60 \mathrm{sec}$. When the mouse failed to locate the platform within $60 \mathrm{sec}$, it was guided to stay on the platform for $10 \mathrm{sec}$. On the fifth day, the platform was removed, and the time spent in the target quadrant and the frequency of crossing through the platform place were recorded.

Western blotting. Brain tissue samples were harvested 2 weeks after NSC transplantation. Tissues were lysed in cold lysis buffer (1X PBS, $1 \%$ Nonidet P-40, $0.5 \%$ sodium deoxycholate and $0.1 \%$ SDS; RIPA) containing proteinase inhibitors. The protein concentration was measured using a bicinchoninic protein assay kit. An equal amount of protein samples $(50 \mu \mathrm{g})$ was loaded in each lane. The protein samples (concentration, $5 \mu \mathrm{g} / \mu \mathrm{l}$; volume, $10 \mu \mathrm{l}$ ) were submitted to $10 \%$ SDS-PAGE prior to electrotransfer on a PVDF membrane. The membrane was incubated in 5\% non-fat milk at room temperature for $1 \mathrm{~h}$ and washed three times with TBST (0.01 M TBS and $0.1 \%$ Tween-20). The specific primary antibodies: Rabbit anti-ChAT $(1: 1,000)$, rabbit anti-Map2 $(1: 1,000)$, rabbit anti-PSD95 $(1: 1,000)$, rabbit anti-SYP $(1: 1,000)$, mouse anti- $\beta$-tubulin III $(1: 1,000)$ were incubated with the membrane overnight at $4^{\circ} \mathrm{C}$. After washing in TBST to remove residual antibodies, horseradish peroxidase (HRP) conjugated secondary antibodies (goat anti-rabbit IgG; 1:2,000 and rabbit anti-mouse 1:1,000) were applied for $2 \mathrm{~h}$ at room temperature. The membrane was then washed with TBST prior to film exposition and development using an ECL kit (Bioworld Technology, Inc.). The protein expression levels were analyzed using ImageJ (version 1.4.3.67; National Institutes of Health).

Immunohistochemistry. Mice were anesthetized and perfused transcardially with saline buffer and a pre-cooled $4 \%$ paraformaldehyde (PFA) solution. The whole brain was removed and fixed overnight in 4\% PFA. Then, dehydration was performed with the use of a sucrose gradient and brains were cut into $30-\mu \mathrm{m}$ thick slices. Slices of basal forebrain and hippocampus were rinsed three times (5 min each time) in PBS and incubated in $0.3 \%$ Triton X-100 to weaken cell membranes. Slices were then incubated in 5\% bovine serum albumin (BSA) at room temperature for $1 \mathrm{~h}$, and ChAT, GFAP and DCX antibodies $(1: 300)$ were added prior to incubation overnight at $4^{\circ} \mathrm{C}$. Alexa Fluor ${ }^{\mathrm{TM}} 594$ donkey anti-rabbit IgG (1:500) was then added after washing the primary antibodies and the samples were incubated at RT for $2 \mathrm{~h}$. DAPI (Wuhan Boster Biological Technology, Ltd.) staining was carried out for $15 \mathrm{~min}$ prior to imaging with the use of a fluorescence microscope (magnification, $\mathrm{x} 200$ and $\mathrm{x} 400$ ).

Statistical analysis. All statistical analyses were computed using the SPSS 16.0 software (SPSS, Inc.). The data were expressed as the mean \pm standard deviation (SD). Multiple group comparisons were achieved by single-effect analysis of variance (one-way ANOVA) followed by post hoc Fisher's LSD multiple comparison test. $\mathrm{P}<0.05$ was considered to indicate a statistically significant difference.

\section{Results}

Mouse EGFP-NSCs have self-renewal and multi-differentiation abilities in vitro. The stem cells used in the present study were produced from 12.5 to 14.5 -day-old mice embryos expressing EGFP. Immunohistochemical analysis revealed co-expression of EGFP and Nestin, a marker of NSCs, in undifferentiated NSCs (Fig. 1A). When incubated with FBS for 5 days, NSCs could differentiate into glial cells and neurons expressing the positive marker GFAP and $\beta$-tubulin III, respectively (Fig. 1B and C).

Survival, differentiation and migration of NSCs in vivo. EGFP-NSCs were prepared to better track the fate of engrafted cells in vivo. Migration and differentiation were observed two weeks after transplantation. As presented in Fig. 2, engrafted cells survived and partly remained at the injection site. Conversely, some cells migrated to surrounding regions including the corpus callosum and the adjacent cortex (Fig. 2A). Some cells experienced morphologic changes. To assess their phenotypes, astrocytes and immature neurons were labeled with GFAP or DCX. Confocal microscopy analysis revealed that a small portion of engrafted cells differentiated into GFAP + astrocytes. Others differentiated into $\mathrm{DCX}+$ neurons (Fig. 2B and C).

NSC transplantation alleviates cognitive impairment. To assess whether NSC transplantation can alleviate cognitive impairment, the MWM was implemented to assay spatial learning and memory ability (Fig. 3A). As anticipated, during the four-day navigation task, shorter latencies could be observed in the three groups. However, this reduction was less pronounced in the Tg-AD group and the latency was significantly shorter in the Tg-NSC group compared to the Tg-AD group on the last trial day (Fig. 3B, $\mathrm{P}<0.05$ ). The crossing times of the original platform were significantly ameliorated in the Tg-NSC group compared to Tg-AD group. However, it was still lower than those observed in the WT group (Fig. 3C, $\mathrm{P}<0.05)$.

NSC transplantation increases the levels of cognitive-related proteins. Synaptic damage is one of the most important pathological features of $\mathrm{AD}$, which is highly correlated with cognitive deficit (14). MAP-2 is overexpressed in dendrites and involved in microtubule assembly. This protein can regulate microtubule networks in axons and neuron dendrites that 


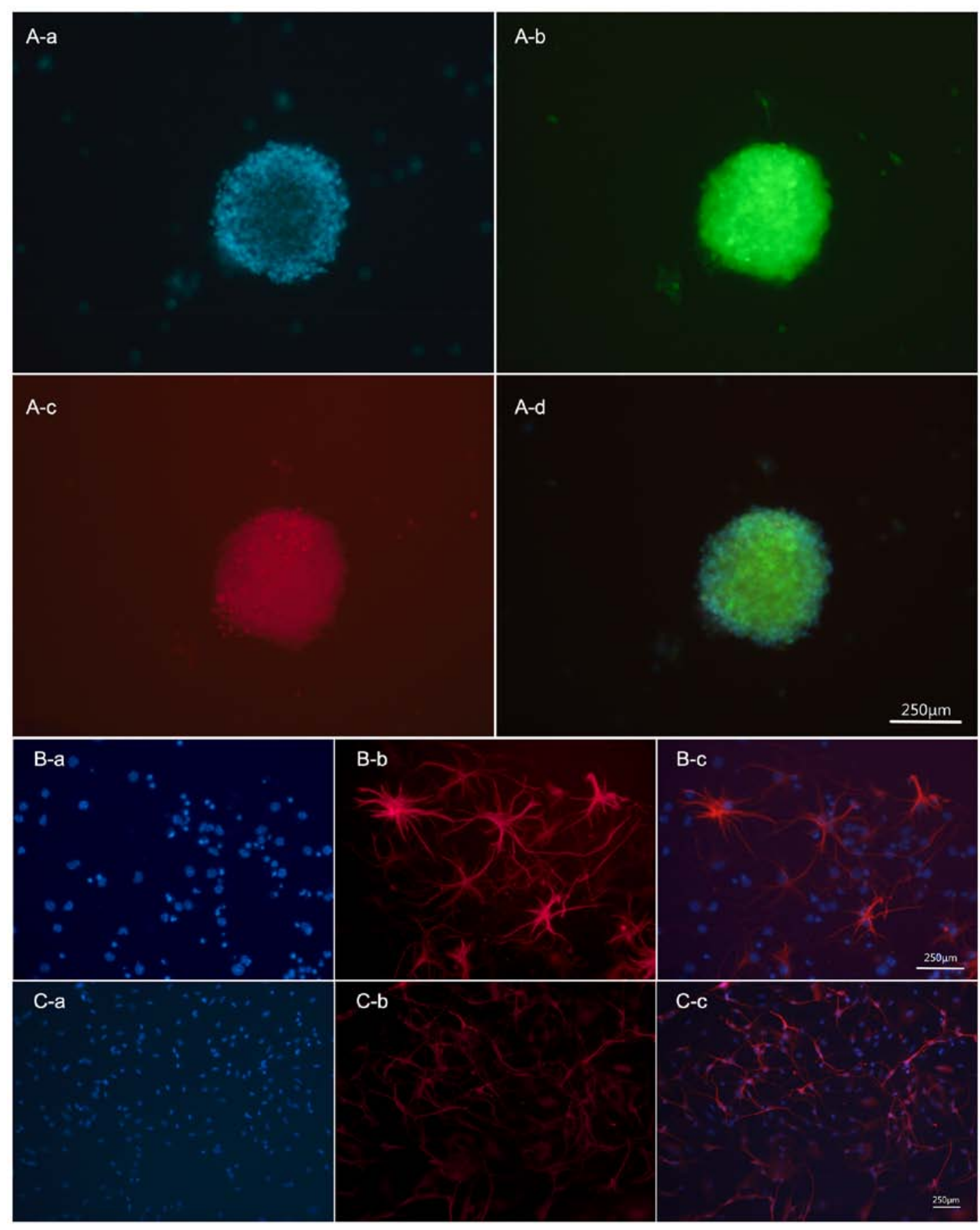

Figure 1. Mouse EGFP-NSCs have the ability of self-renewal and multi-differentiation in vitro. (A) Cultured NSCs were amassed as neurospheres and co-expressed EGFP (green) and Nestin (red). Subpart A-d is a merge image, shows DAPI (blue fluorescence, A-a) + EGFP (green fluorescence, A-b) + Nestin (red fluorescence, A-c). (B) NSCs differentiated into astrocyte, expression of GFAP-positive markers (red). Subpart B-c is a merge image, shows DAPI (blue fluorescence, B-a) + GFAP (red fluorescence, B-b). (C) NSCs differentiated into neurons, expression of Tuj1-positive markers (red). Subpart C-c is a merge image, shows DAPI (blue fluorescence, C-a) + Tuj1 (red fluorescence, C-b). Scale bar, $100 \mu \mathrm{m}$. EGFP, enhanced green fluorescent protein; NSCs, neural stem cells.

may be critical for neurogenesis (15). In the present study, the levels of SYP, PSD-95 and MAP-2 proteins were significantly increased in the Tg-NSC group compared with the Tg-AD group and no difference could be observed in comparison with the WT group (Fig. 4, P<0.05).

NSC transplantation protects cholinergic neurons in the basal forebrain. In order to determine whether NSC transplantation restored atrophic cholinergic neurons in the AD mouse model, ChAT-positive neurons and ChAT protein expression were analyzed. The results are displayed in Fig. 5. NSC transplantation markedly increased the levels of ChAT proteins and ChAT-positive neurons in the basal forebrain. There were significant differences compared with the control group (Tg-AD). However, ChAT protein levels were still lower compared to the WT group (Fig. 5A and B). NSC transplantation increased ChAT protein levels in the basal forebrain, but not in the hippocampus (Fig. 5C). 

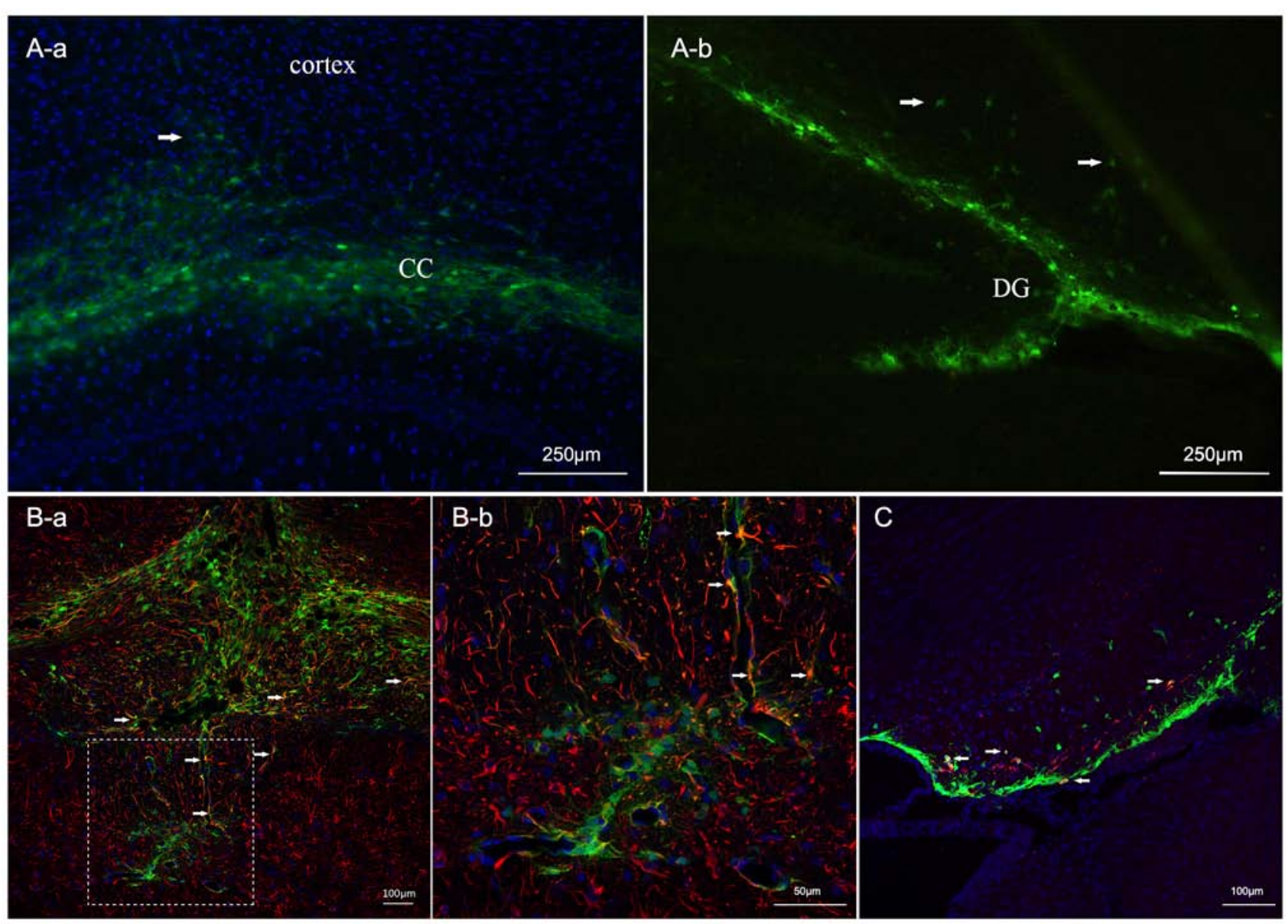

Figure 2. The fate of NSCs two weeks after transplantation to the hippocampus. (A) Engrafted cells migrated to ambient regions including the corpus callosum, adjacent cortex, and deeper in the hippocampus. A-a shows EGFP-NSCs migrated to the corpus callosum and the adjacent cortex (as indicated by the arrow). A-b shows the cells migrated in the hippocampus (the arrows show EGFP-NSCs). (B) Engrafted cells differentiated into GFAP + astrocytes (as indicated by the arrow); subpart B-a is the injection site, B-b is the partial magnification of the marked area in B-a. (C) Certain engrafted cells differentiated into DCX + neurons (as indicated by the arrow). NSCs, neural stem cells.

\section{Discussion}

In the present study, MWM was used to detect hippocampal-dependent learning and memory. Furthermore, confocal microscopy was applied to investigate the migration and differentiation of transplanted cells and ChAT-positive neurons. Western blotting was performed to assess the levels of SYP, PSD-95, MAP-2 and ChAT proteins in the hippocampus and the basal forebrain. These analyses revealed that engrafted cells survived, migrated and differentiated in the brains of AD mice. Moreover, learning and memory ability were rescued by NSC transplantation. The mechanism of NSC transplantation may be related to the restoration of the synaptic impairment and the cholinergic neuron recovery in the basal forebrain.

APP/PS1 transgenic mice are useful in studying neurological brain disorders and have been widely used to mimic the pathology of AD. Mice develop significant impairment after the age of nine months. In the present study, 12-month-old APP/PS1 mice were used. It was revealed that Tg-AD mice exhibited cognitive defects and that the learning ability was significantly increased after NSC transplantation. However, it was still lower than that of the corresponding WT control mice.
Previous studies revealed that the accumulation of pathological $A \beta$ proteins causes synaptic loss related with AD-associated cognitive deficit (16). Moreover, synaptic loss is the principal correlate of the loss of cholinergic neurons (2). Thus, in the present study, several important protein markers associated with the synaptic plasticity were selected. SYP is a marker of presynaptic proteins. SYP k.o. mice exhibited retarded learning with slightly impaired memory performance (14). PSD-95 is another important synaptic protein. This protein is a constituent of the postsynaptic complex and plays an important role in synaptic plasticity and the stabilization of synaptic changes during long-term potentiation (17). In the present study, it was revealed that the protein levels of SYP and PSD-95 significantly increased in the hippocampus of the Tg-NSC group compared to the Tg-AD group. Accordingly, the cognitive function was also improved. In addition, MAP-2 protein levels were also increased in the hippocampus after NSC transplantation. The present results indicated that NSC transplantation can restore synaptic impairment in accordance with previous research.

Another important typical feature of AD is the damage of cholinergic neurons in the basal forebrain (18). Evidence has revealed that cholinergic neurons from the medial septum and the diagonal band of Broca (MSDB), within the basal 
A a

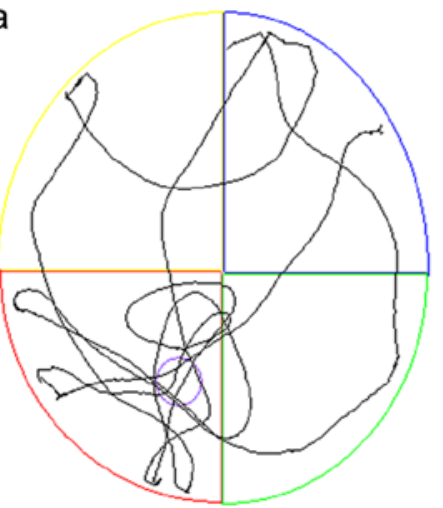

WT b

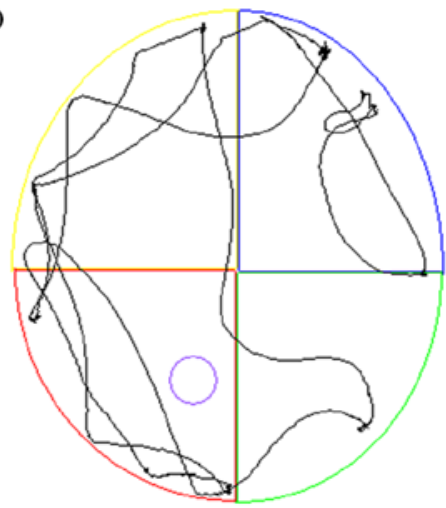

Tg-AD

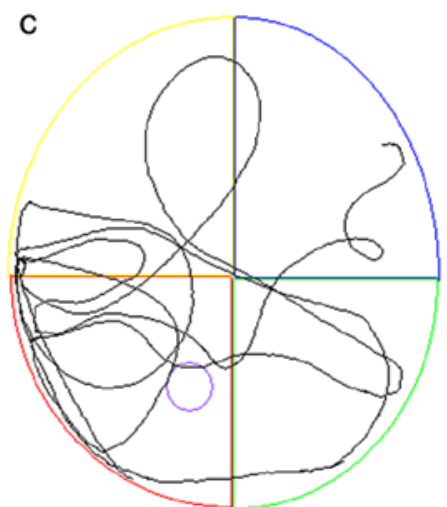

Tg-NSC
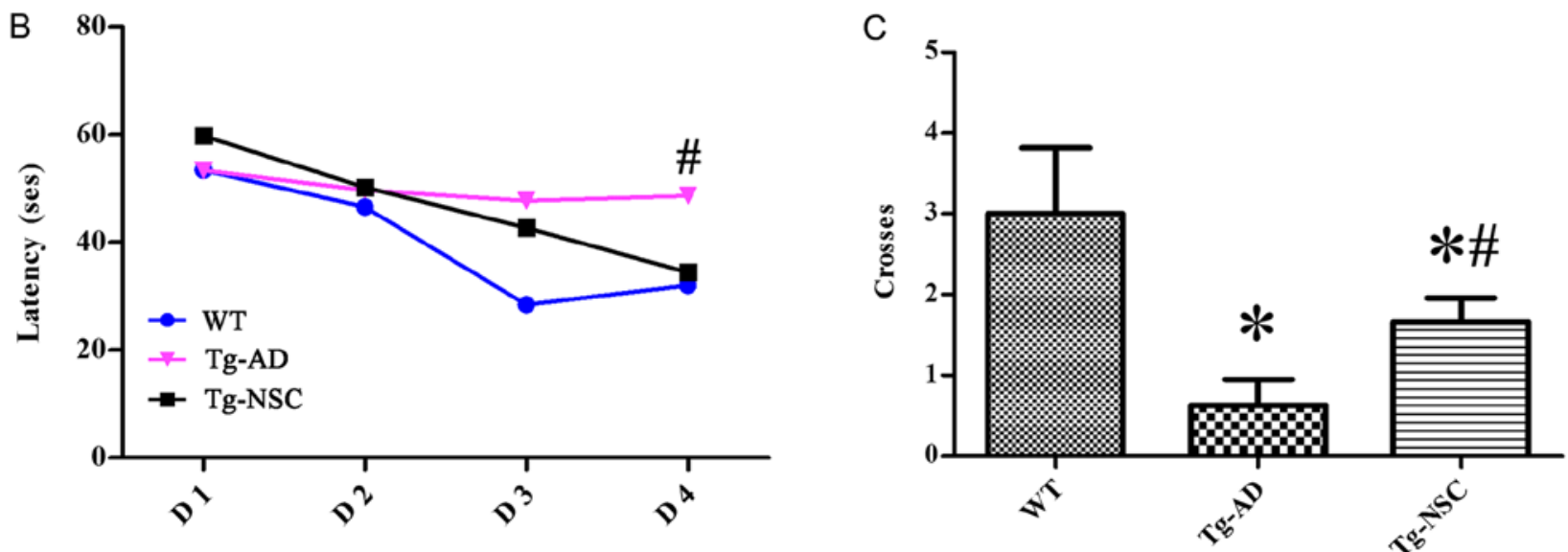

Figure 3. MWM test performances two weeks after NSC transplantation. (A) Representative movement traces from 3 groups on the day of exploration. The Tg-mice exhibited more dispersed paths, suggesting memory impairments; the Tg-NSC and WT groups had slightly concentrated swimming paths. A-a, A-b and A-c show the WT, Tg-AD and Tg-NSc groups, respectively. (B) Time latency in a navigation test. All animals exhibited improvements in finding the platform. (C) Crossing times of the original platform. "P<0.05 compared with WT group; ${ }^{*} \mathrm{P}<0.05$ compared with Tg-AD group. MWM, Morris water-maze; NSC, neural stem cell; Tg, transgenic; AD, Alzheimer's disease; WT, wild-type.

A

SYP

PSD-95

WT Tg-AD Tg-NSC
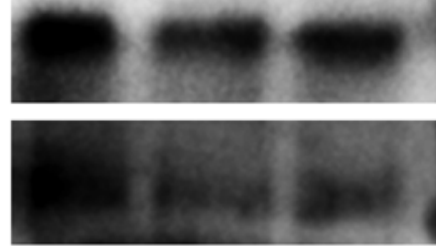

Tubulin

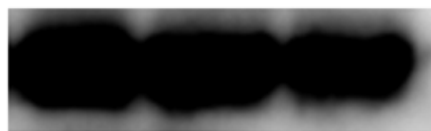

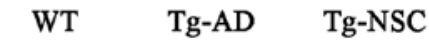

2

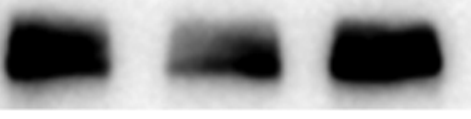

Tubulin

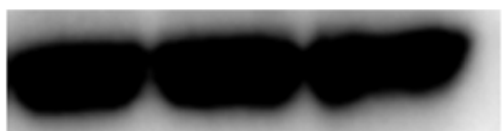

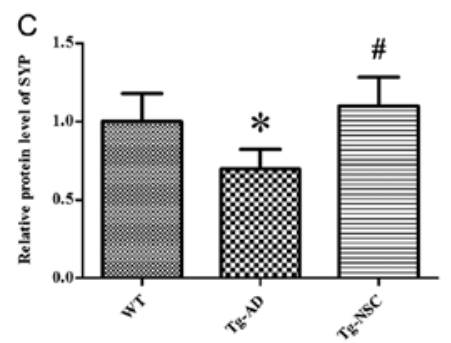
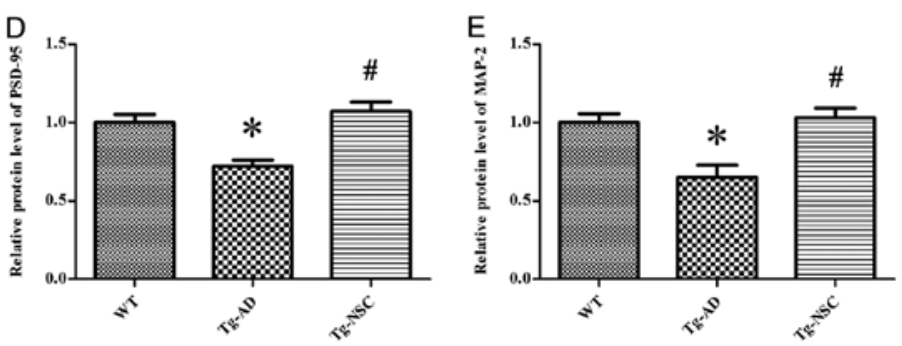

Figure 4. Cognitive-related protein expression in the hippocampus. (A and B) The protein levels of SYP, PSD-95 and MAP-2 in the hippocampus after NSC transplantation. (C-E) Relative levels of SYP, PSD-95 and MAP-2 protein. ${ }^{*} \mathrm{P}<0.05$ compared with WT group; ${ }^{\text {} P}<0.05$ compared with Tg-AD group. SYP, synaptophysin; PSD-95, postsynaptic density protein 95; MAP-2, microtubule-associated protein; NSC, neural stem cell; WT, wild-type; Tg, transgenic; $\mathrm{AD}$, Alzheimer's disease. 

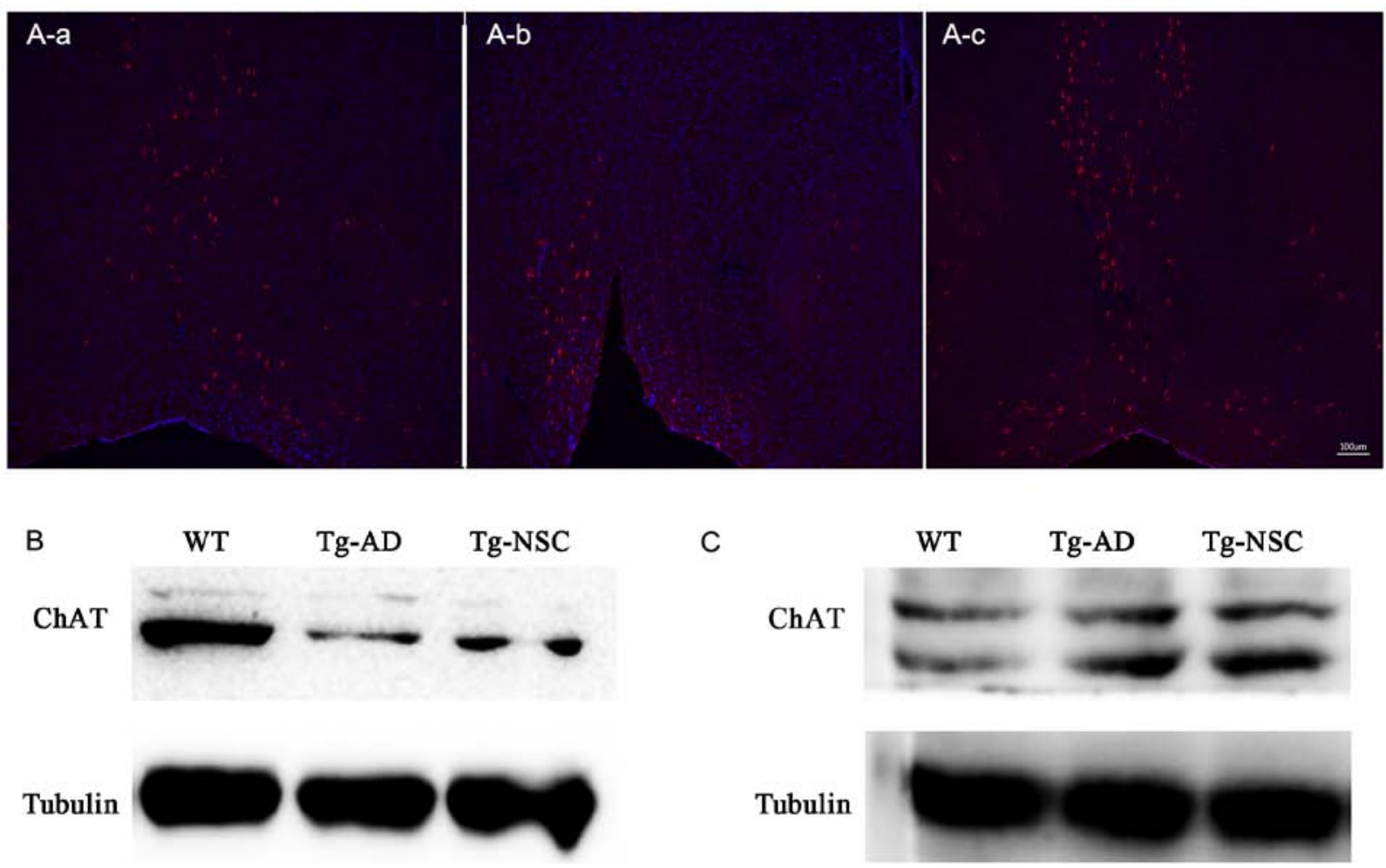

Basal forebrain

Hippocampus
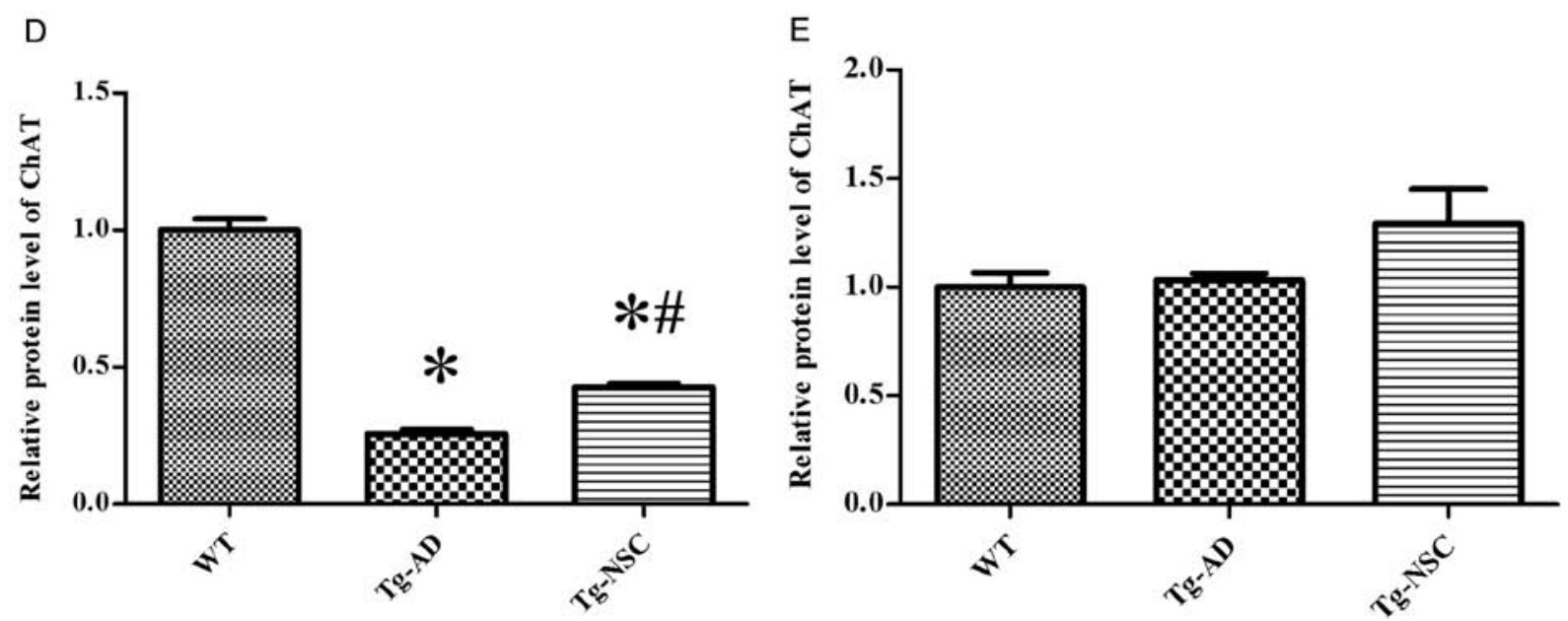

Figure 5. Cholinergic neurons in the basal forebrain. (A) Confocal display the ChAT-positive neurons, (A-a) WT group; (A-b) Tg-AD group; (A-c) Tg-NSC group. (B and C) ChAT protein expression in the basal forebrain and hippocampus. (D and E) Relative levels of ChAT protein in the basal forebrain and hippocampus. "P $<0.05$ compared with WT group; ${ }^{\text {}} \mathrm{P}<0.05$ compared with Tg-AD group. ChAT, choline acetyltransferase; WT, wild-type; Tg, transgenic; $\mathrm{AD}$, Alzheimer's disease; NSC, neural stem cell.

forebrain projections $(\sim 65 \%)$ to the hippocampus, provide the main source of acetylcholine in the hippocampus (19). Cholinergic hypofunction induces the loss of cortical and hippocampus cholinergic innervation and may cause cognitive disorders. In the present study, NSCs were transplanted into the basal forebrain and hippocampus of an $\mathrm{AD}$ rat model with basal forebrain lesion. Damaged neurons were restored and replenished (P75 was used as a marker of cholinergic neurons), and learning and memory abilities were improved (20). It was revealed that NSC transplantation increased the levels of ChAT protein in the basal forebrain, but not in the hippocampus. Moreover, immunofluorescence analysis demonstrated that ChAT-positive neurons were restored in the Tg-NSC group with a significant improvement compared to the Tg-AD group. Moreover, no difference could be observed in comparison with the WT group. These results were inconsistent with previous research revealing that dysfunction of cholinergic neurons, rather than cholinergic cell loss, is impacted. In this study, the number of ChAT-positive neurons was unchanged, however, trkA and p75NTR-containing neurons, which co-localize with ChAT, were significantly reduced in the nucleus basalis (21).

Numerous studies have revealed that NSC transplantation alleviates learning and memory impairment in AD mouse 
models. The possible mechanism may be related to the engrafted cell itself or to the secretion or play a bystander role by transplanted cells $(11 ; 22)$. In the present study, the recovery of injured synapses and atrophic cholinergic neurons in the basal forebrain were observed. However, interactions between these improved pathologies remain poorly defined. Transplanted NSCs survived, migrated, and differentiated into GFAP and DCX-positive neural cells. This differentiation was limited. It was assumed that neuronal replacement by grafted cells is not possible. Some studies have suggested that it is challenging that transplanted cells could differentiate into target neurons and then be projected to the appropriate region to form appropriate synaptic connections (9). The beneficial effects that were observed in this AD model may be explained by cell complement or only protection rather than replacement. The recovery of cholinergic functions is most likely due to NSCs themselves, which can release neurotrophic factors $(22,23)$, and be uptaken by axonal terminals and retrogradely be transported into the cell body. These factors protect basal forebrain cholinergic neurons (24). Notably, although cholinergic fiber projections to the hippocampus decreased in $\mathrm{AD}$, ChAT protein levels increased in the hippocampus. Similar to previous studies, it can be suggested that the upregulation of hippocampal ChAT in MCI cases may be due to the replacement of denervated glutamatergic synapses by cholinergic input arising from the septum (25). Collectively, the present data indicated that AD transgenic mice display cognitive impairment associated with damage or loss of cholinergic neurons in the basal forebrain, and that cell transplantation may enhance ChAT protein levels and restore cholinergic neurons.

In conclusion, the present study revealed that NSC transplantation can protect basal forebrain cholinergic neurons and restore synaptic impairment as well as eventually improve learning and memory functions in an APP/PS1 transgenic (Tg) mouse model.

\section{Acknowledgements}

Not applicable.

\section{Funding}

The present study was supported by The Natural Science Foundation of Guangdong Province (2016A030310275).

\section{Availability of data and materials}

The datasets used during the present study are available from the corresponding author upon reasonable request.

\section{Authors' contributions}

QZ performed experiments, and collected and interpreted the data. NZ interpreted the data and drafted the manuscript. $\mathrm{NH}$ and RJ collected the data and performed the statistical analysis. HL fed the animals and performed the experiments. AX searched the literature, analyzed the data and revised the manuscript. DL designed the study and searched the literature. YC designed the study and revised the manuscript.

\section{Ethics approval and consent to participate}

All procedures were approved by The Animal Ethics Committee of the Second Affiliated Hospital of Guangzhou Medical University (approval no. A2018-016).

\section{Patient consent for publication}

Not applicable.

\section{Competing interests}

The authors declare that they have no competing interest.

\section{References}

1. Anand R, Gill KD and Mahdi AA: Therapeutics of Alzheimer's disease: Past, present and future. Neuropharmacology 76: 27-50, 2014.

2. Cattaneo A and Calissano P: Nerve growth factor and Alzheimer's disease: New facts for an old hypothesis. Mol Neurobiol 46: 588-604, 2012

3. Small G and Bullock R: Defining optimal treatment with cholinesterase inhibitors in Alzheimer's disease. Alzheimers Dement 7: 177-184, 2011.

4. Teng YD: Functional multipotency of stem cells: Biological traits gleaned from neural progeny studies. Semin Cell Dev Biol: pii: S1084-S9521, 2019 (Epub ahead of print).

5. Li XY, Bao XJ and Wang RZ: Potential of neural stem cell-based therapies for Alzheimer's disease. J Neurosci Res 93: 1313-1324, 2015.

6. Alipour M, Nabavi SM, Arab L, Vosough M, Pakdaman H, Ehsani E and Shahpasand K: Stem cell therapy in Alzheimer's disease: Possible benefits and limiting drawbacks. Mol Biol Rep 46: 1425-1446, 2019.

7. Blurton-Jones M, Spencer B, Michael S, Castello NA, Agazaryan AA, Davis JL, Müller FJ, Loring JF, Masliah E and LaFerla FM: Neural stem cells genetically-modified to express neprilysin reduce pathology in Alzheimer transgenic models. Stem Cell Res Ther 5: 46, 2014.

8. Zhang W, Gu GJ, Zhang Q, Liu JH, Zhang B, Guo Y, Wang MY, Gong QY and Xu JR: NSCs promote hippocampal neurogenesis, metabolic changes and synaptogenesis in APP/PS1 transgenic mice. Hippocampus 27: 1250-1263, 2017.

9. Marsh SE and Blurton-Jones M: Neural stem cell therapy for neurodegenerative disorders: The role of neurotrophic support. Neurochem Int 106: 94-100, 2017.

10. Zhang W, Gu GJ, Shen X, Zhang Q, Wang GM and Wang PJ: Neural stem cell transplantation enhances mitochondrial biogenesis in a transgenic mouse model of Alzheimer's disease-like pathology. Neurobiol Aging 36: 1282-1292, 2015.

11. Tang J: How close is the stem cell cure to the Alzheimer's disease: Future and beyond? Neural Regen Res 7: 66-71, 2012.

12. Whitehouse PJ, Price DL, Struble RG, Clark AW, Coyle JT and Delon MR: Alzheimer's disease and senile dementia: Loss of neurons in the basal forebrain. Science 215: 1237-1239, 1982.

13. Müller C and Remy S: Septo-hippocampal interaction. Cell Tissue Res 373: 565-575, 2018.

14. Schmitt U, Tanimoto N, Seeliger M, Schaeffel F and Leube RE: Detection of behavioral alterations and learning deficits in mice lacking synaptophysin. Neuroscience 162: 234-243, 2009.

15. Dehmelt L and Halpain S: The MAP2/Tau family of microtubuleassociated proteins. Genome Biol 6: 204, 2005.

16. Shankar GM, Li S, Mehta TH, Garcia-Munoz A, Shepardson NE, Smith I, Brett FM, Farrell MA, Rowan MJ, Lemere CA, et al: Amyloid-beta protein dimers isolated directly from Alzheimer's brains impair synaptic plasticity and memory. Nat Med 14: 837-842, 2008

17. Tu S, Okamoto S, Lipton SA and Xu H: Oligomeric A $\beta$-induced synaptic dysfunction in Alzheimer's disease. Mol Neurodegener 9: $48,2014$.

18. Hampel H, Mesulam MM, Cuello AC, Farlow MR, Giacobini E, Grossberg GT, Khachaturian AS, Vergallo A, Cavedo E, Snyder PJ and Khachaturian ZS: The cholinergic system in the pathophysiology and treatment of Alzheimer's disease. Brain 141: 1917-1933, 2018. 
19. Sun Y, Nguyen AQ, Nguyen JP, Le L, Saur D, Choi J, Callaway EM and $\mathrm{Xu}$ X: Cell-type-specific circuit connectivity of hippocampal CA1 revealed through Cre-dependent rabies tracing. Cell Rep 7: 269-280, 2014.

20. Chen Y, Pan C, Xuan A, Xu L, Bao G, Liu F, Fang J and Long D: Treatment efficacy of NGF nanoparticles combining neural stem cell transplantation on Alzheimer's disease model rats. Med Sci Monit 21: 3608-3615, 2015.

21. Mufson EJ, Counts SE, Fahnestock M and Ginsberg SD: Cholinotrophic molecular substrates of mild cognitive impairment in the elderly. Curr Alzheimer Res 4: 340-350, 2007.

22. Lu P, Jones LL, Snyder EY and Tuszynski MH: Neural stem cells constitutively secrete neurotrophic factors and promote extensive host axonal growth after spinal cord injury. Exp Neurol 181: 115-129, 2003.

23. Li B, Gao Y, Zhang W and Xu JR: Regulation and effects of neurotrophic factors after neural stem cell transplantation in a transgenic mouse model of Alzheimer disease. J Neurosci Res 96: 828-840, 2018.
24. Conner JM, Franks KM, Titterness AK, Russell K, Merrill DA, Christie BR, Sejnowski TJ and Tuszynski MH: NGF is essential for hippocampal plasticity and learning. J Neurosci 29: 10883-10889, 2009.

25. Mufson EJ, Counts SE, Perez SE and Ginsberg SD: Cholinergic system during the progression of Alzheimer's disease: Therapeutic implications. Expert Rev Neurother 8: 1703-1718, 2008.

(i) (9) This work is licensed under a Creative Commons Attribution-NonCommercial-NoDerivatives 4.0 International (CC BY-NC-ND 4.0) License. 